\title{
HARDY SPACES ESTIMATES FOR MULTILINEAR OPERATORS WITH HOMOGENEOUS KERNELS
}

\author{
YONG DING AND SHANZHEN LU
}

\begin{abstract}
In this paper the authors prove that a class of multilinear operators formed by the singular integral or fractional integral operators with homogeneous kernels are bounded operators from the product spaces $L^{p_{1}} \times$ $L^{p_{2}} \times \cdots \times L^{p_{K}}\left(\mathbb{R}^{n}\right)$ to the Hardy spaces $H^{q}\left(\mathbb{R}^{n}\right)$ and the weak Hardy space $H^{q, \infty}\left(\mathbb{R}^{n}\right)$, where the kernel functions $\Omega_{i j}$ satisfy only the $L^{s}$-Dini conditions. As an application of this result, we obtain the $\left(L^{p}, L^{q}\right)$ boundedness for a class of commutator of the fractional integral with homogeneous kernels and BMO function.
\end{abstract}

\section{$\S 1$. Introduction and statements of results}

It is known that the Jacobin determinant $J(f, g)$ of the functions pair $(f, g)$ is defined by $J(f, g)=\frac{\partial f}{\partial x_{1}} \frac{\partial g}{\partial x_{2}}-\frac{\partial f}{\partial x_{2}} \frac{\partial g}{\partial x_{1}}$. Lions and Meyer proved that $J$ is bounded from the product space $L_{1}^{2} \times L_{1}^{2}$ of the Sobolev spaces to the Hardy space $H^{1}\left(\mathbb{R}^{n}\right)$. Because of its importance in the harmonic analysis and partial differential equations, it has been studied by some authors in recent years. In 1992, Coifman and Grafakos extended the above result to give the mapping properties on the Hardy space for a class of the multilinear operator formed by the Calderón-Zygmund singular integrals [CG], [G]. Recently, Miyachi [Mi] studied the similar mapping properties for a class of the multilinear operator formed by the Calderón-Zygmund singular integrals and the Riesz potential operators. This is an extension of Coifman-Grafakos's results. In [DL4], we also obtained the same conclusion as in $[\mathrm{G}]$ but under a weaker condition.

In this paper, we will use the $L^{p}$ boundedness of the singular integral with rough kernel and the $\left(L^{p}, L^{q}\right)$ boundedness of rough fractional integral operator to prove that the multilinear operator formed by the products

Received September 5, 2000.

2000 Mathematics Subject Classification: 42B25, 47G10.

The research was supported by National Natural Science Foundation (Grant: 10271016), Doctoral Programme Foundation of Institution of Higher Education (Grant: 20010027002) and National 973 Project of China. 
of the singular integral or fractional integral operators with homogeneous kernels are bounded from the product space $L^{p_{1}} \times L^{p_{2}} \times \cdots \times L^{p_{K}}\left(\mathbb{R}^{n}\right)$ into the Hardy spaces $H^{q}\left(\mathbb{R}^{n}\right)$ and the weak Hardy space $H^{q, \infty}\left(\mathbb{R}^{n}\right)$. In our results, we need only that the kernel function $\Omega$ to satisfy $L^{s}$-Dini condition, which is weaker than the smoothness condition assumed in $[\mathrm{CG}],[\mathrm{G}]$ and [Mi], respectively.

Now let us give some definitions. Suppose that $S^{n-1}$ is the unit sphere of $\mathbb{R}^{n}(n \geq 2)$ equipped with normalized Lebesgue measure $d \sigma\left(x^{\prime}\right)$ and $\Omega \in$ $L^{1}\left(S^{n-1}\right)$ is homogeneous of degree zero on $\mathbb{R}^{n}$. Then the singular integral and fractional integral operators with homogeneous kernel are respectively defined by

$$
T_{\Omega} f(x)=p \cdot v \cdot \int_{\mathbb{R}^{n}} \frac{\Omega(x-y)}{|x-y|^{n}} f(y) d y
$$

and

$$
T_{\Omega, \alpha} f(x)=\int_{\mathbb{R}^{n}} \frac{\Omega(x-y)}{|x-y|^{n-\alpha}} f(y) d y,
$$

where $0<\alpha<n$. Obviously, when $\Omega \equiv 1$ the operator $T_{\Omega, \alpha}$ is just the Riesz potential operator. See [CWW], [MW], [DL1]-[DL3] and [D] for the boundedness of $T_{\Omega, \alpha}$ on the various spaces (or weighted spaces).

We say that $\Omega$ satisfies the $L^{s}$-Dini condition $(s \geq 1)$ if $\Omega$ is homogeneous of degree zero on $\mathbb{R}^{n}$ with $\Omega \in L^{s}\left(S^{n-1}\right)$ and

$$
\int_{0}^{1} \omega_{s}(\delta) \frac{d \delta}{\delta}<\infty
$$

where $\omega_{s}(\delta)$ denotes the integral modulus of continuity of order $s$ of $\Omega$ defined by

$$
\omega_{s}(\delta)=\sup _{\|\rho\|<\delta}\left(\int_{S^{n-1}}\left|\Omega\left(\rho x^{\prime}\right)-\Omega\left(x^{\prime}\right)\right|^{s} d \sigma\left(x^{\prime}\right)\right)^{1 / s}
$$

and $\rho$ is a rotation on $S^{n-1}$ with $\|\rho\|=\sup _{x^{\prime} \in S^{n-1}}\left|\rho x^{\prime}-x^{\prime}\right|$.

Throughout this article, $N$ and $K$ will denote fixed integers satisfying $K, N \geq 2$. It is said that $r$ is the harmonic mean of $p_{1}, p_{2}, \ldots, p_{K}>1$ if $1 / r=1 / p_{1}+1 / p_{2}+\cdots+1 / p_{K}$. Moreover, for $i=1,2, \ldots, N ; j=1,2, \ldots, K$,

(i) $0 \leq \alpha_{i j}<n / p_{j}$ and $\alpha:=\sum_{j=1}^{K} \alpha_{i j} \geq 0(i=1,2, \ldots, N)$; 
(ii) $\Omega_{i j}$ satisfies $L^{s_{i j}}$-Dini condition with $s_{i j} \geq 1$.

(iii) If $\alpha_{i j}=0$, then

$$
\int_{S^{n-1}} \Omega_{i j}\left(x^{\prime}\right) d \sigma\left(x^{\prime}\right)=0 .
$$

Now we define the $K$-linear operator by

$$
L_{\Omega, \alpha}(\vec{f})(x)=\sum_{i=1}^{N}\left[T_{\Omega_{i 1}, \alpha_{i 1}} f_{1}(x)\right]\left[T_{\Omega_{i 2}, \alpha_{i 2}} f_{2}(x)\right] \cdots\left[T_{\Omega_{i K}, \alpha_{i K}} f_{K}(x)\right],
$$

where $\vec{f}=\left(f_{1}, f_{2}, \ldots, f_{K}\right)$ and $T_{\Omega_{i j}, \alpha_{i j}}$ defined in (1.2). When $\alpha_{i j}=0$, $T_{\Omega_{i j}, \alpha_{i j}}$ becomes indeed into the singular integral operator $T_{\Omega_{i j}}$ defined in (1.1). In this case, we need $\Omega_{i j}$ to satisfy (1.3).

Let us now formulate our main result as follows. In Theorem 1, the multilinear operator $L_{\Omega, \alpha}$ is formed by the fractional integral operators $T_{\Omega_{i j}, \alpha_{i j}}$ or the singular integral operators $T_{\Omega_{i j}}$.

TheOREm 1. Suppose that $r$ is the harmonic mean of $p_{1}, p_{2}, \ldots, p_{K}>$ 1. For $i=1,2, \ldots, N$ and $j=1,2, \ldots, K, \alpha_{i j} \geq 0$ satisfy (i) and $\Omega_{i j}$ satisfies (ii) with $s_{i j}>p_{j}^{\prime}$ and (iii), respectively. Moreover, $0 \leq \alpha<n-1$ and $1 / q=1 / r-\alpha / n$. If the harmonic mean of any proper subset of the set $\left\{p_{1}, p_{2}, \ldots, p_{K}\right\}$ is greater than one and for all $\vec{f} \in\left(C_{0}^{\infty}\right)^{K}$,

$$
\int x^{\beta} L_{\Omega, \alpha}(\vec{f})(x) d x=0 \quad \text { for }|\beta| \leq m,
$$

where $m$ satisfies $0 \leq m<n-1-\alpha$. Then we have the following conclusions:

(a) When $\frac{n}{n+m+1+\alpha}<r \leq \frac{n}{n+\alpha}$ (equivalently $\frac{n}{n+m+1}<q \leq 1$ ), $L_{\Omega, \alpha}$ can be extended into a bounded mapping from $L^{p_{1}} \times L^{p_{2}} \times \cdots \times L^{p_{K}}$ to $H^{q}$.

(b) When $r=\frac{n}{n+m+1+\alpha}$ (equivalently $\left.q=\frac{n}{n+m+1}\right), L_{\Omega, \alpha}$ can be extended into a bounded mapping from $L^{p_{1}} \times L^{p_{2}} \times \cdots \times L^{p_{K}}$ to $H^{q, \infty}$.

Remark 1. If $\alpha=m=0$, then the Theorem 1 is just the main conclusion obtained in [DL4]. Thus Theorem 1 generalized the main theorem in [DL4] in two ways, i.e., $\alpha \geq 0$ and $m \geq 0$.

As a direct application of Theorem 1, we prove that the commutator $T_{\Omega, \alpha, b}$ formed by the homogeneous fractional integral operator $T_{\Omega, \alpha}$ Gand a function $b(x)$ in $B M O$ is bounded from $L^{p}\left(\mathbb{R}^{n}\right)$ to $L^{q}\left(\mathbb{R}^{n}\right)$. This is an extension of Chanillo's famous result on commutator of the Riesz potential operator [Cha]. 
TheOREM 2. Suppose that $0<\alpha<n-1,1<p<n / \alpha$ and $1 / q=$ $1 / p-\alpha / n$. If $\Omega \in L^{s}\left(S^{n-1}\right)\left(s>\max \left\{p^{\prime}, q\right\}\right)$ satisfying $L^{s}$-Dini condition, then there is a constant $C>0$, independent of $f$, such that

$$
\left\|T_{\Omega, \alpha, b}(f)\right\|_{q} \leq C\|b\|_{B M O}\|f\|_{p}
$$

where the commutator $T_{\Omega, \alpha, b}$ is defined by $T_{\Omega, \alpha, b}(f)(x)=b(x) T_{\Omega, \alpha} f(x)-$ $T_{\Omega, \alpha}(b f)(x)$.

\section{§2. Some elementary results and lemmas}

In this section let us recall some known results and give some lemmas which will be used in the proofs of our theorems.

Theorem A. ([DL3]) Suppose that $0<\alpha<n, 1<p<n / \alpha$ and $1 / q=1 / p-\alpha / n$. If $\Omega \in L^{s}\left(S^{n-1}\right)(s>n /(n-\alpha))$ to be homogeneous of degree zero on $\mathbb{R}^{n}$, then $T_{\Omega, \alpha}$ is bounded operator from $L^{p}\left(\mathbb{R}^{n}\right)$ to $L^{q}\left(\mathbb{R}^{n}\right)$.

Theorem B. ([CZ], [Che]) Let $\Omega \in L^{s}\left(S^{n-1}\right)(s>1)$ to be homogeneous of degree zero on $\mathbb{R}^{n}$ and satisfy (1.3). Then for $1<p<\infty$ there exists a $C>0$, independent of $f$, such that

$$
\left\|T_{\Omega} f\right\|_{p} \leq C\|f\|_{p} \quad \text { and } \quad\left\|T_{\Omega}^{*} f\right\|_{p} \leq C\|f\|_{p},
$$

where $T_{\Omega}^{*}$ denotes the maximal operator of $T_{\Omega}$ defined by

$$
T_{\Omega}^{*} f(x)=\sup _{\varepsilon>0}\left|\int_{|x-y| \geq \varepsilon} \frac{\Omega(x-y)}{|x-y|^{n}} f(y) d y\right| .
$$

Lemma 1. ([KW]) Suppose that $\Omega$ satisfies the $L^{s}$-Dini condition $(s>$ $1)$. If there is a constant $a_{0}$ with $0<a_{0}<1 / 2$ such that $|x|<a_{0} R$, then

$$
\begin{aligned}
& \left(\int_{R<|y|<2 R}\left|\frac{\Omega(y-x)}{|y-x|^{n}}-\frac{\Omega(y)}{|y|^{n}}\right|^{s} d y\right)^{1 / s} \\
& \quad \leq C R^{n / s-n}\left\{\frac{|x|}{R}+\int_{|x| / 2 R<\delta<|x| / R} \omega_{s}(\delta) \frac{d \delta}{\delta}\right\}
\end{aligned}
$$

where the constant $C$ is independent of $R$ and $x$. 
Lemma 2. Suppose that $\Omega$ satisfies the $L^{s}$-Dini condition for $s>1$. Then there is a $C>0$, i ndependent of $f$, such that for any $x_{0} \in \mathbb{R}^{n}, t>0$ and any $x$ with $\left|x-x_{0}\right| \leq t$,

$$
\left|\int_{\left|y-x_{0}\right| \geq 2 t}\left(\frac{\Omega(x-y)}{|x-y|^{n}}-\frac{\Omega\left(x_{0}-y\right)}{\left|x_{0}-y\right|^{n}}\right) f(y) d y\right| \leq C\left[M\left(|f|^{s^{\prime}}\right)\left(x_{0}\right)\right]^{1 / s^{\prime}}
$$

where $M$ denotes the Hardy-Littlewood maximal operator defined by

$$
M f(x)=\sup _{r>0} \frac{1}{r^{n}} \int_{|x-y|<r}|f(y)| d y \text {. }
$$

In fact, by Hölder's inequality and Lemma 1 we have

$$
\begin{aligned}
& \left|\int_{\left|y-x_{0}\right| \geq 2 t}\left(\frac{\Omega(x-y)}{|x-y|^{n}}-\frac{\Omega\left(x_{0}-y\right)}{\left|x_{0}-y\right|^{n}}\right) f(y) d y\right| \\
& \leq \sum_{j=1}^{\infty}\left(\int_{2^{j} t \leq\left|y-x_{0}\right|<2^{j+1} t}\left|\frac{\Omega(x-y)}{|x-y|^{n}}-\frac{\Omega\left(x_{0}-y\right)}{\left|x_{0}-y\right|^{n}}\right|^{s} d y\right)^{1 / s} \\
& \quad \times\left(\int_{\left|y-x_{0}\right|<2^{j+1} t}|f(y)|^{s^{\prime}} d y\right)^{1 / s^{\prime}} \\
& \leq C \sum_{j=1}^{\infty}\left(\frac{1}{2^{j}}+\int_{\left|x-x_{0}\right| / 2^{j+1} t}^{\left|x-x_{0}\right| / 2^{j} t} \omega_{s}(\delta) \frac{d \delta}{\delta}\right)\left(\frac{1}{\left(2^{j+1} t\right)^{n}} \int_{\left|y-x_{0}\right|<2^{j+1} t}|f(y)|^{s^{\prime}} d y\right)^{1 / s^{\prime}} \\
& \leq C\left[M\left(|f|^{s^{\prime}}\right)\left(x_{0}\right)\right]^{1 / s^{\prime}}\left(1+\int_{0}^{1} \omega_{s}(\delta) \frac{d \delta}{\delta}\right) \leq C\left[M\left(|f|^{s^{\prime}}\right)\left(x_{0}\right)\right]^{1 / s^{\prime}} .
\end{aligned}
$$

LEMma 3. ([DL3]) Suppose that $0<\alpha<n$ and $\Omega$ satisfies the $L^{s}$-Dini condition with $s>1$. If there is a constant $a_{0}$ with $0<a_{0}<1 / 2$ such that $|x|<a_{0} R$, then

$$
\begin{aligned}
& \left(\int_{R<|y|<2 R}\left|\frac{\Omega(y-x)}{|y-x|^{n-\alpha}}-\frac{\Omega(y)}{|y|^{n-\alpha}}\right|^{s} d y\right)^{1 / s} \\
& \quad \leq C R^{n / s-(n-\alpha)}\left\{\frac{|x|}{R}+\int_{|x| / 2 R<\delta<|x| / R} \frac{\omega_{r}(\delta)}{\delta} d \delta\right\}
\end{aligned}
$$

where the constant $C>0$ is independent of $R$ and $x$. 
Lemma 4. Suppose that $0<\alpha<n$ and $\Omega$ satisfies the $L^{s}$-Dini condition with $s>1$. Then there is a $C>0$, independent of $f$, such that for any $x_{0} \in \mathbb{R}^{n}, t>0$ and any $x$ with $\left|x-x_{0}\right| \leq t$,

$$
\left|\int_{\left|y-x_{0}\right| \geq 2 t}\left(\frac{\Omega(x-y)}{|x-y|^{n-\alpha}}-\frac{\Omega\left(x_{0}-y\right)}{\left|x_{0}-y\right|^{n-\alpha}}\right) f(y) d y\right| \leq C\left[M_{\alpha s^{\prime}}\left(|f|^{s^{\prime}}\right)\left(x_{0}\right)\right]^{1 / s^{\prime}}
$$

where $M_{\lambda}$ denotes the fractional maximal operator defined by

$$
M_{\lambda} f(x)=\sup _{r>0} \frac{1}{r^{n-\lambda}} \int_{|x-y|<r}|f(y)| d y \quad \text { for } 0<\lambda<n .
$$

By Lemma 3 and using the same method in proving Lemma 2, we may get Lemma 4.

Lemma 5. Suppose that $0<\alpha<n, 1<p<n / \alpha$ and $1 / r=1 / p-$ $\alpha / n$. If $1 \leq s^{\prime}<p$, then

$$
\left\|\left[M_{\alpha s^{\prime}}\left(|f|^{s^{\prime}}\right)(\cdot)\right]^{1 / s^{\prime}}\right\|_{r} \leq C\|f\|_{p}
$$

In fact, this is a direct result of the $\left(L^{p}, L^{r}\right)$ boundedness the fractional maximal operator $M_{\lambda}$ (see [T], for example).

\section{§3. Proof of Theorem 1}

First let us consider the case of $\alpha_{i j}>0$ for all $i, j$. In the proof of Theorem 1 we use some idea from $[\mathrm{G}]$. Take $\phi \in C_{0}^{\infty}\left(\mathbb{R}^{n}\right)$ such that $\operatorname{supp}(\phi) \subset$ $\left\{x \in \mathbb{R}^{n}:|x| \leq 1\right\}$. For $x, x_{0} \in \mathbb{R}^{n}$ we define $\phi_{t, x_{0}}(x)=\frac{1}{t^{n}} \phi\left(\frac{x-x_{0}}{t}\right)$. By the maximal function characterization of the Hardy spaces and weak Hardy spaces (see $[\mathrm{S}]$ and $[\mathrm{FS}]$, or $[\mathrm{Lu}]$ ), we need to verify that

$$
\sup _{t>0}\left|\int \phi_{t,(\cdot)}(x) L_{\Omega, \alpha}(\vec{f})(x) d x\right| \in \begin{cases}L^{q}\left(\mathbb{R}^{n}\right), & \text { for } n /(n+m+1)<q \leq 1 \\ L^{q, \infty}\left(\mathbb{R}^{n}\right), & \text { for } q=n /(n+m+1) .\end{cases}
$$

Fix a smooth cut-off $\eta(x)$ such that $\eta(x) \geq 0$ and $\eta(x) \equiv 1$ on $|x|<2$, $\eta(x)=0$ on $|x| \geq 4$. Let $\eta_{0}(x)=\eta\left(\frac{x-x_{0}}{t}\right)$ and $\eta_{1}(x)=1-\eta_{0}(x)$. By expanding the following equality

$$
L_{\Omega, \alpha}\left(\eta_{0} f_{1}, \ldots, \eta_{0} f_{K}\right)=L_{\Omega, \alpha}\left(f_{1}-\eta_{1} f_{1}, \ldots, f_{K}-\eta_{1} f_{K}\right),
$$


and solving out $L_{\Omega, \alpha}(\vec{f})$, we may get $L_{\Omega, \alpha}(\vec{f})(x)=L_{0}+L_{1}+\cdots+L_{K}$, where

$$
\begin{aligned}
L_{0} & =L_{\Omega, \alpha}\left(\eta_{0} f_{1}, \eta_{0} f_{2}, \ldots, \eta_{0} f_{K}\right) \\
L_{1} & =\sum_{j=1}^{K} L_{\Omega, \alpha}\left(f_{1}, \ldots, \eta_{1} f_{j}, \ldots, f_{K}\right) \\
L_{2} & =-\sum_{1 \leq j_{1}<j_{2} \leq K} L_{\Omega, \alpha}\left(f_{1}, \ldots, \eta_{1} f_{j_{1}}, \ldots, \eta_{1} f_{j_{2}}, \ldots, f_{K}\right) \\
& \left.\ldots \ldots \ldots, \eta_{1} f_{j_{1}}, \ldots, \eta_{1} f_{j_{l}}, \ldots, f_{K}\right) \\
L_{l} & =(-1)^{l+1} \sum_{1 \leq j_{1}<j_{2}<\cdots<j_{l} \leq K} L_{\Omega, \alpha}\left(f_{1}, \ldots, \eta_{1} f_{j_{1}}, \ldots, \eta_{1} f_{j_{2}}, \ldots, \eta_{1} f_{K}\right) . \\
& \ldots \ldots \ldots \\
L_{K} & =(-1)^{K+1} L_{\Omega, \alpha}\left(\eta_{1} f_{1}, \eta_{1} f_{2}, \ldots\right.
\end{aligned}
$$

To prove (3.1), it suffices to show for $0 \leq l \leq K$

$$
\sup _{t>0}\left|\int \phi_{t,(\cdot)}(x) L_{l}(x) d x\right| \in L^{q}\left(\mathbb{R}^{n}\right), \quad \text { if } n /(n+m+1)<q \leq 1,
$$

and

$$
\begin{aligned}
\left|\left\{x_{0} \in \mathbb{R}^{n}: \sup _{t>0}\left|\int \phi_{t, x_{0}}(x) L_{l}(x) d x\right|>\lambda\right\}\right| \leq & \frac{C}{\lambda^{q}} \prod_{j=1}^{K}\left\|f_{j}\right\|_{p_{j}}^{q}, \\
& \text { if } q=n /(n+m+1),
\end{aligned}
$$

where $C>0$ is independent of $\lambda$ and $\vec{f}$.

Let us begin by giving the estimate for the term $L_{1}$. Note that

$$
\begin{aligned}
& \left|L_{1}(x)\right| \leq \sum_{j=1}^{K}\left(\sum _ { i = 1 } ^ { N } | T _ { \Omega _ { i 1 } , \alpha _ { i 1 } } f _ { 1 } ( x ) | \cdots \left[\left|T_{\Omega_{i j}, \alpha_{i j}}\left(\eta_{1} f_{j}\right)(x)-T_{\Omega_{i j}, \alpha_{i j}}\left(\eta_{1} f_{j}\right)\left(x_{0}\right)\right|\right.\right. \\
& \left.\left.\quad+\left|T_{\Omega_{i j}, \alpha_{i j}}\left(\eta_{1} f_{j}\right)\left(x_{0}\right)\right|\right] \cdots\left|T_{\Omega_{i K}, \alpha_{i K}} f_{K}(x)\right|\right),
\end{aligned}
$$

and by Lemmas 3 and 4 if $\left|x-x_{0}\right| \leq t, \Omega \in L^{s^{\prime}}\left(S^{n-1}\right)$ then

$$
\begin{aligned}
& \sup _{t>0}\left|T_{\Omega, \alpha}\left(\eta_{1} f\right)\left(x_{0}\right)\right| \leq \sup _{t>0} \int_{\left|x_{0}-y\right| \geq 2 t} \frac{\left|\Omega\left(x_{0}-y\right)\right|}{\left|x_{0}-y\right|^{n-\alpha}}|f(y)| d y \leq T_{|\Omega|, \alpha}(|f|)\left(x_{0}\right), \\
& \sup _{t>0}\left|T_{\Omega, \alpha}\left(\eta_{1} f\right)(x)-T_{\Omega, \alpha}\left(\eta_{1} f\right)\left(x_{0}\right)\right| \leq C\left[M_{\alpha s^{\prime}}\left(|f|^{s^{\prime}}\right)\left(x_{0}\right)\right]^{1 / s^{\prime}} .
\end{aligned}
$$


Thus we get

$$
\begin{aligned}
& \sup _{t>0}\left|\int \phi_{t, x_{0}}(x) L_{1}(x) d x\right| \\
& \leq \sum_{l=1}^{K} \sum_{i=1}^{N} \sup _{t>0} \int\left|\phi_{t, x_{0}}(x)\right| \prod_{\substack{1 \leq j \leq K \\
j \neq l}}\left|T_{\Omega_{i j}, \alpha_{i j}} f_{j}(x)\right| \\
& \quad \times\left(\left|T_{\Omega_{i l}, \alpha_{i l}}\left(\eta_{1} f_{l}\right)(x)-T_{\Omega_{i l}, \alpha_{i l}}\left(\eta_{1} f_{l}\right)\left(x_{0}\right)\right|+\left|T_{\Omega_{i l}, \alpha_{i l}}\left(\eta_{1} f_{l}\right)\left(x_{0}\right)\right|\right) d x \\
& \leq C \sum_{l=1}^{K} \sum_{i=1}^{N}\left(\prod_{\substack{1 \leq j \leq K \\
j \neq l}}\left|T_{\Omega_{i j}, \alpha_{i j}} f_{j}\right|\right)^{*}\left(x_{0}\right) \\
& \quad \times\left(\left[M_{\alpha_{i l}} s_{i l}^{\prime}\left(\left|f_{l}\right|^{s_{i l}^{\prime}}\right)\left(x_{0}\right)\right]^{1 / s_{i l}^{\prime}}+T_{\left|\Omega_{i l}\right|, \alpha_{i l}}\left(f_{l}\right)\left(x_{0}\right)\right)
\end{aligned}
$$

where we denote the Hardy-Littlewood maximal function of $g$ at $x_{0}$ by $g^{*}\left(x_{0}\right)$. Let $1 / r_{i j}=1 / p_{j}-\alpha_{i j} / n$ for $1 \leq j \leq K$. If taking $1 / \sigma_{i l}=$ $\sum_{\substack{1 \leq j \leq K \\ j \neq l}} 1 / r_{i j}$, then we have $1 / q=1 / \sigma_{i l}+1 / r_{i j}$. Since the harmonic mean of any proper subset of the $p_{j}^{\prime}$ s is greater than one, we have

$$
1 / \sigma_{i l}=\sum_{\substack{1 \leq j \leq K \\ j \neq l}}\left(1 / p_{j}-\alpha_{i j} / n\right)<\sum_{\substack{1 \leq j \leq K \\ j \neq l}} 1 / p_{j}<1 .
$$

Thus by Hölder's inequality, Theorem A and the $L^{p}$-boundedness of the Hardy-Littlewood maximal function and Lemma 5 (note that $s_{i l}^{\prime}<p_{l}$ ), the $L^{q}$ norm in $x_{0}$ of the last term in (3.5) is bounded by

$$
\begin{gathered}
C \sum_{l=1}^{K} \sum_{i=1}^{N}\left\|\left[M_{\alpha_{i l} s_{i l}^{\prime}}\left(\left|f_{l}\right|^{s_{i l}^{\prime}}\right)(\cdot)\right]^{1 / s_{i l}^{\prime}}+T_{\left|\Omega_{i l}\right|, \alpha_{i l}}\left(\left|f_{l}\right|\right)(\cdot)\right\|_{r_{i l}} \\
\times\left\|\left(\prod_{\substack{1 \leq j \leq K \\
j \neq l}}\left|T_{\Omega_{i j}, \alpha_{i l}} f_{j}\right|\right)^{*}\right\|_{\sigma_{i l}} \\
\leq C \sum_{l=1}^{K} \sum_{i=1}^{N}\left(\|\left[M_{\alpha_{i l} s_{i l}^{\prime}}\left(\left|f_{l}\right|^{\left.s_{i l}^{\prime}\right)^{1 / s_{i l}^{\prime}} \|_{r_{i l}}}+\left\|T_{\left|\Omega_{i l}\right|, \alpha_{i l}}\left(\left|f_{l}\right|\right)\right\|_{r_{i l}}\right)\right.\right. \\
\times \prod_{\substack{1 \leq j \leq K \\
j \neq l}} T_{\Omega_{i j}, \alpha_{i l}}\left(f_{j}\right) \|_{\sigma_{i l}}
\end{gathered}
$$




$$
\begin{aligned}
& \leq C \sum_{l=1}^{K} \sum_{i=1}^{N}\left\|f_{l}\right\|_{p_{l}} \prod_{\substack{1 \leq j \leq K \\
j \neq l}}\left\|T_{\Omega_{i j}, \alpha_{i l}}\left(f_{j}\right)\right\|_{r_{i l}} \\
& \leq C \sum_{l=1}^{K}\left\|f_{l}\right\|_{p_{l}} \prod_{\substack{1 \leq j \leq K \\
j \neq l}}\left\|f_{j}\right\|_{p_{j}} \leq C \prod_{j=1}^{K}\left\|f_{j}\right\|_{p_{j}} .
\end{aligned}
$$

Hence

$$
\left\|\sup _{t>0}\left|\int \phi_{t,(\cdot)}(x) L_{1}(x) d x\right|\right\|_{q} \leq C \prod_{j=1}^{K}\left\|f_{j}\right\|_{p_{j}} .
$$

From the proof (3.6) it is easy to see that (3.6) holds indeed for $n /(n+m+$ $1) \leq q \leq 1$. Thus for $q=n /(n+m+1)$ we can get the following inequality by $(3.6)$

$$
\left|\left\{x_{0} \in \mathbb{R}^{n}: \sup _{t>0}\left|\int \phi_{t, x_{0}}(x) L_{1}(x) d x\right|>\lambda\right\}\right| \leq \frac{C}{\lambda^{q}} \prod_{j=1}^{K}\left\|f_{j}\right\|_{p_{j}}^{q},
$$

where $C$ is independent of $\lambda$ and $\vec{f}$.

Term $L_{2}$ is treated similarly. We write $L_{2}=L_{21}+L_{22}+L_{23}+L_{24}$, where

$$
\begin{gathered}
L_{21}=-\sum_{1 \leq j_{1}<j_{2} \leq K}\left(\sum_{i=1}^{N}\left[T_{\Omega_{i 1}, \alpha_{i 1}} f_{1}(x)\right] \cdots\right. \\
\cdots\left[T_{\Omega_{i j_{1}}, \alpha_{i j_{1}}}\left(\eta_{1} f_{j_{1}}\right)(x)-T_{\Omega_{i j_{1}}, \alpha_{i j_{1}}}\left(\eta_{1} f_{j_{1}}\right)\left(x_{0}\right)\right] \cdots \\
\cdots\left[T_{\Omega_{i j_{2}}, \alpha_{i j_{2}}}\left(\eta_{1} f_{j_{2}}\right)(x)-T_{\Omega_{i j_{2}}, \alpha_{i j_{2}}}\left(\eta_{1} f_{j_{2}}\right)\left(x_{0}\right)\right] \cdots \\
\left.\cdots\left[T_{\Omega_{i K}, \alpha_{i K}} f_{K}(x)\right]\right) \\
L_{22}=\sum_{1 \leq j_{1}<j_{2} \leq K}\left(\sum_{i=1}^{N}\left[T_{\Omega_{i 1}, \alpha_{i 1}} f_{1}(x)\right] \cdots\left[T_{\Omega_{i j_{1}}, \alpha_{i j_{1}}}\left(\eta_{1} f_{j_{1}}\right)\left(x_{0}\right)\right] \cdots\right. \\
\cdots\left[T_{\Omega_{i j_{2}}, \alpha_{i j_{2}}}\left(\eta_{1} f_{j_{2}}\right)(x)-T_{\Omega_{i j_{2}}, \alpha_{i j_{2}}}\left(\eta_{1} f_{j_{2}}\right)\left(x_{0}\right)\right] \cdots \\
\left.\cdots\left[T_{\Omega_{i K}, \alpha_{i K}} f_{K}(x)\right]\right) \\
\sum_{1 \leq j_{1}<j_{2} \leq K}\left(\sum_{i=1}^{N}\left[T_{\Omega_{i 1}, \alpha_{i 1}} f_{1}(x)\right] \cdots\right.
\end{gathered}
$$




$$
\begin{array}{r}
\cdots\left[T_{\Omega_{i j_{1}}, \alpha_{i j_{1}}}\left(\eta_{1} f_{j_{1}}\right)(x)-T_{\Omega_{i j_{1}}, \alpha_{i j_{1}}}\left(\eta_{1} f_{j_{1}}\right)\left(x_{0}\right)\right] \cdots \\
\left.\cdots\left[T_{\Omega_{i j_{2}}, \alpha_{i j_{2}}}\left(\eta_{1} f_{j_{2}}\right)\left(x_{0}\right)\right] \cdots\left[T_{\Omega_{i K}, \alpha_{i K}} f_{K}(x)\right]\right) \\
L_{24}=-\sum_{1 \leq j_{1}<j_{2} \leq K}\left(\sum_{i=1}^{N}\left[T_{\Omega_{i 1}, \alpha_{i 1}} f_{1}(x)\right] \cdots\left[T_{\Omega_{i j_{1}}, \alpha_{i j_{1}}}\left(\eta_{1} f_{j_{1}}\right)\left(x_{0}\right)\right] \cdots\right. \\
\left.\cdots\left[T_{\Omega_{i j_{2}}, \alpha_{i j_{2}}}\left(\eta_{1} f_{j_{2}}\right)\left(x_{0}\right)\right] \cdots\left[T_{\Omega_{i K}, \alpha_{i K}} f_{K}(x)\right]\right) .
\end{array}
$$

By Lemma 4 and (3.4) we may show that any term $L_{2 u}(u=1,2,3,4)$ satisfy the following estimate

$$
\begin{gathered}
\sup _{t>0}\left|\int \phi_{t, x_{0}}(x) L_{2 u}(x) d x\right| \leq C \sum_{\substack{1 \leq j_{1}<j_{2} \leq K \\
i=1}}^{N}\left(\prod_{\substack{1 \leq l \leq K \\
l \neq j_{1}, j_{2}}}\left|T_{\Omega_{i l}, \alpha_{i l}} f_{l}\right|\right)^{*}\left(x_{0}\right) \\
\times C_{i j_{1}}\left(f_{j_{1}}\right)\left(x_{0}\right) C_{i j_{2}}\left(f_{j_{2}}\right)\left(x_{0}\right) .
\end{gathered}
$$

By (3.4), we know that each $C_{i j_{m}}\left(f_{j_{m}}\right)(m=1,2)$ is either

$$
\left[M_{\alpha_{i j_{m}} s_{i j_{m}}^{\prime}}\left(\left|f_{j_{m}}\right|^{s_{i j_{m}}^{\prime}}\right)\right]^{1 / s_{i j_{m}}^{\prime}} \quad \text { or } \quad T_{\left|\Omega_{i j_{m}}\right|, \alpha_{i j_{m}}}\left(\left|f_{j_{m}}\right|\right) \text {. }
$$

By Theorem A and Lemma 5 (note that $s_{i j_{m}}^{\prime}<p_{j_{m}}$ ), we have $\left\|C_{i j_{m}}\left(f_{j_{m}}\right)\right\|_{r_{i j_{m}}} \leq C\left\|f_{j_{m}}\right\|_{p_{j_{m}}}$. Now define $\sigma_{i v}$ by $1 / q=1 / r_{i j_{1}}+1 / r_{i j_{2}}+$ $1 / \sigma_{i v}$. From the conditions of Theorem 1 we know that $\sigma_{i v}>1$. Using the same method treated $L_{1}$, for $u=1,2,3,4$, we get

$$
\begin{aligned}
& \left\|\sup _{t>0}\left|\int \phi_{t,(\cdot)}(x) L_{2 u}(x) d x\right|\right\|_{q} \\
& \quad \leq C \sum_{1 \leq j_{1}<j_{2} \leq K} \sum_{i=1}^{N}\left\|\left(\prod_{\substack{1 \leq l \leq K \\
l \neq j_{1}, j_{2}}}\left|T_{\Omega_{i l}, \alpha_{i l}} f_{l}\right|\right)^{*}\right\|\left\|_{\sigma_{i v}}\right\| C_{i j_{1}}\left(f_{j_{1}}\right)\left\|_{r_{i j_{1}}}\right\| C_{i j_{2}}\left(f_{j_{2}}\right) \|_{r_{i j_{2}}} \\
& \quad \leq C \sum_{1 \leq j_{1}<j_{2} \leq K} \sum_{i=1}^{N}\left\|\prod_{\substack{1 \leq l \leq K \\
l \neq j_{1}, j_{2}}} T_{\Omega_{i l}, \alpha_{i l}} f_{l}\right\|_{\sigma_{i v}}\left\|f_{j_{1}}\right\|_{p_{1}}\left\|f_{j_{2}}\right\|_{p_{j_{2}}} \\
& \quad \leq C \sum_{1 \leq j_{1}<j_{2} \leq K} \sum_{i=1}^{N}\left(\prod_{\substack{1 \leq l \leq K \\
l \neq j_{1}, j_{2}}}\left\|f_{l}\right\|_{p_{l}}\right)\left\|f_{j_{1}}\right\|_{p_{j_{1}}}\left\|f_{j_{2}}\right\|_{p_{j_{2}}} \leq C \prod_{1 \leq l \leq K}\left\|f_{l}\right\|_{p_{l} .} .
\end{aligned}
$$


Since (3.8) holds for $n /(n+m+1) \leq q \leq 1$, we have

$$
\left|\left\{x_{0} \in \mathbb{R}^{n}: \sup _{t>0}\left|\int \phi_{t, x_{0}}(x) L_{2}(x) d x\right|>\lambda\right\}\right| \leq \frac{C}{\lambda^{q}} \prod_{j=1}^{K}\left\|f_{j}\right\|_{p_{j}}^{q} .
$$

where $q=n /(n+m+1)$ and $C$ is independent of $\lambda$ and $\vec{f}$.

Applying the method treated $L_{1}$ and $L_{2}$, we may prove that (3.2) and (3.3) hold also for the terms $L_{3}, L_{4}, \ldots, L_{K}$. Here we omit the details. Thus, to complete the proof of Theorem 1, it remains to verify that (3.2) and (3.3) hold still for $L_{0}$. In order to do this, we need the following lemma.

LEMMA 6. Under the conditions of Theorem 1, we have

(i) if $n /(n+m+1+\alpha)<r \leq n /(n+\alpha)$, then there exists $1<d_{j}<p_{j}$, $(1 \leq j \leq K)$ such that

$$
\sup _{t>0}\left|\int \phi_{t, x_{0}}(x) L_{0}(x) d x\right| \leq C \prod_{j=1}^{K}\left[M_{\alpha_{i j} d_{j}}\left(\left|f_{j}\right|^{d_{j}}\right)\left(x_{0}\right)\right]^{1 / d_{j}}
$$

(ii) if $r=n /(n+m+1+\alpha)$, then

$$
\sup _{t>0}\left|\int \phi_{t, x_{0}}(x) L_{0}(x) d x\right| \leq C \prod_{j=1}^{K}\left[M_{\alpha_{i j} p_{j}}\left(\left|f_{j}\right|^{p_{j}}\right)\left(x_{0}\right)\right]^{1 / p_{j}} .
$$

Proof. First we give the proof of (3.11). By the definition of $L_{0}(x)$ and the moment condition (1.4), we have

$$
\begin{aligned}
& \left|\int \phi_{t, x_{0}}(x) L_{0}(x) d x\right| \\
& =\mid \sum_{i=1}^{N} \int\left[\phi_{t, x_{0}}(x)-\sum_{|\beta| \leq m} \frac{1}{\beta !} \phi_{t, x_{0}}^{(\beta)}\left(y_{1}\right)\left(x-y_{1}\right)^{\beta}\right] \\
& \quad \times \int \frac{\Omega_{i 1}\left(x-y_{1}\right)}{\left|x-y_{1}\right|^{n-\alpha_{i 1}}}\left(\eta_{0} f_{1}\right)\left(y_{1}\right) d y_{1} \prod_{j=2}^{K} T_{\Omega_{i j}, \alpha_{i j}}\left(\eta_{0} f_{j}\right)(x) d x \mid \\
& \leq \sum_{i=1}^{N} \iint\left|\phi_{t, x_{0}}(x)-\sum_{|\beta| \leq m} \frac{1}{\beta !} \phi_{t, x_{0}}^{(\beta)}\left(y_{1}\right)\left(x-y_{1}\right)^{\beta}\right| \\
& \quad \times \frac{\left|\Omega_{i 1}\left(x-y_{1}\right)\right|}{\left|x-y_{1}\right|^{n-\alpha_{i 1}}\left|\left(\eta_{0} f_{1}\right)\left(y_{1}\right)\right| d y_{1} \prod_{j=2}^{K}\left|T_{\Omega_{i j}, \alpha_{i j}}\left(\eta_{0} f_{j}\right)(x)\right| d x .}
\end{aligned}
$$


Since

$$
\left|\phi_{t, x_{0}}(x)-\sum_{|\beta| \leq m} \frac{1}{\beta !} \phi_{t, x_{0}}^{(\beta)}\left(y_{1}\right)\left(x-y_{1}\right)^{\beta}\right| \leq C t^{-n-m-1}\left|x-y_{1}\right|^{m+1},
$$

we have

$$
\begin{aligned}
& \int\left|\phi_{t, x_{0}}(x)-\sum_{|\beta| \leq m} \frac{1}{\beta !} \phi_{t, x_{0}}^{(\beta)}\left(y_{1}\right)\left(x-y_{1}\right)^{\beta}\right| \frac{\left|\Omega_{i 1}\left(x-y_{1}\right)\right|}{\left|x-y_{1}\right|^{n-\alpha_{i 1}}}\left|\left(\eta_{0} f_{1}\right)\left(y_{1}\right)\right| d y_{1} \\
& \quad \leq C \int t^{-n-m-1}\left|x-y_{1}\right|^{m+1} \frac{\left|\Omega_{i 1}\left(x-y_{1}\right)\right|}{\left|x-y_{1}\right|^{n-\alpha_{i 1}}}\left|\left(\eta_{0} f_{1}\right)\left(y_{1}\right)\right| d y_{1} \\
& \quad=C t^{-n-m-1} T_{\left|\Omega_{i 1}\right|, m+1+\alpha_{i 1}}\left(\left|\eta_{0} f_{1}\right|\right)(x) .
\end{aligned}
$$

Since $\sum_{j=1}^{K} 1 / p_{j}=(n+m+1+\alpha) / n$ and $\sum_{j=2}^{K} 1 / p_{j}<1$, we have $1 / p_{1}>$ $\left(m+1+\alpha_{i 1}\right) / n$. Denote $1 / \sigma_{i 1}=1 / p_{1}-\left(m+1+\alpha_{i 1}\right) / n$ and $1 / r_{i j}=$ $1 / p_{j}-\alpha_{i j} / n$ for $j=2, \ldots, K$, then by (3.12), (3.13) and applying Hölder's inequality and Theorem $\mathrm{A}$ we have

$$
\begin{aligned}
& \sup _{t>0}\left|\int \phi_{t, x_{0}}(x) L_{0}(x) d x\right| \\
& \quad \leq C \sup _{t>0} \sum_{i=1}^{N} t^{-n-m-1}\left\|T_{\left|\Omega_{i 1}\right|, m+1+\alpha_{i 1}}\left(\left|\eta_{0} f_{1}\right|\right)\right\|_{\sigma_{i 1}} \prod_{j=2}^{K}\left\|T_{\left|\Omega_{i j}\right|, \alpha_{i j}}\left(\left|\eta_{0} f_{j}\right|\right)\right\|_{r_{i j}} \\
& \quad \leq C \sup _{t>0} t^{-n-m-1}\left\|\eta_{0} f_{1}\right\|_{p_{1}} \prod_{j=2}^{K}\left\|\eta_{0} f_{j}\right\|_{p_{j}} \\
& \quad \leq C \prod_{j=1}^{K}\left[M_{\alpha_{i j} p_{j}}\left(\left|f_{j}\right|^{p_{j}}\right)\left(x_{0}\right)\right]^{1 / p_{j}}
\end{aligned}
$$

where we use the assumption $\alpha=\sum_{j=1}^{K} \alpha_{i j}$ for all $i=1,2, \ldots, N$. This is just (3.11). Let us now consider (3.10). First we show that by

$$
1 / p_{1}+\cdots+1 / p_{K}=1 / r<(n+m+1+\alpha) / n
$$

we can choose some $d_{j}(1 \leq j \leq K)$ such that

(a) $1<d_{j}<p_{j}$ for $1 \leq j \leq K$;

(b) $1 / d_{2}+\cdots+1 / d_{K}<1$; 
(c) $1 / d_{1}+\cdots+1 / d_{K}=(n+m+1+\alpha) / n$.

In fact, under the conditions of Theorem 1 , it is easy to see that $\max \left\{1 / p_{2}+\cdots+1 / p_{K},(m+1+\alpha) / n\right\}<\min \left\{1,1 / p_{1}^{\prime}+(m+1+\alpha) / n\right\}$.

Thus, we can choose $\delta$ such that

$\max \left\{1 / p_{2}+\cdots+1 / p_{K},(m+1+\alpha) / n\right\}<\delta<\min \left\{1,1 / p_{1}^{\prime}+(m+1+\alpha) / n\right\}$.

Taking $\varepsilon_{2}, \ldots, \varepsilon_{K}>0$, such that

$$
\left(1+\varepsilon_{2}\right) / p_{2}+\cdots+\left(1+\varepsilon_{K}\right) / p_{K}=\delta .
$$

Obviously, for $2 \leq j \leq K$ we have $1+\varepsilon_{j}<p_{j}$. Let

$$
\varepsilon=(n+m+1+\alpha) / n-\left(1 / p_{1}+\cdots+1 / p_{K}\right)>0 .
$$

Then by (3.14) we know that $\varepsilon>\varepsilon_{2} / p_{2}+\cdots+\varepsilon_{K} / p_{K}$. Denote

$$
\varepsilon_{1}=\left[\varepsilon-\left(\varepsilon_{2} / p_{2}+\cdots+\varepsilon_{K} / p_{K}\right)\right] p_{1}>0,
$$

then

$(n+m+1+\alpha) / n=\varepsilon+1 / p_{1}+\cdots+1 / p_{K}=\left(1+\varepsilon_{1}\right) / p_{1}+\cdots+\left(1+\varepsilon_{K}\right) / p_{K}$ and $1+\varepsilon_{1}<p_{1}$. In fact, by $(m+1+\alpha) / n<\delta$, we have

$$
\begin{aligned}
1+\varepsilon_{1} & =\left[\varepsilon-\left(\varepsilon_{2} / p_{2}+\cdots+\varepsilon_{K} / p_{K}\right)\right] p_{1}+1 \\
& =\left[\frac{n+m+1+\alpha}{n}-\frac{1}{p_{1}}-\left(\frac{1+\varepsilon_{2}}{p_{2}}+\cdots+\frac{1+\varepsilon_{K}}{p_{K}}\right)\right] p_{1}+1 \\
& =\left[1 / p_{1}^{\prime}+(1+\alpha) / n-\delta\right] p_{1}+1<p_{1} / p_{1}^{\prime}+1=p_{1} .
\end{aligned}
$$

Now set $d_{j}=p_{j} /\left(1+\varepsilon_{j}\right), 1 \leq j \leq K$, then $d_{j}{ }^{\prime} s$ satisfy (a), (b) and (c). Thus by the conclusion of (3.11), we obtain (3.10). Thus we complete the proof of Lemma 6 .

Below we use Lemma 6 to prove that (3.2) and (3.3) hold for $L_{0}$. First let us consider (3.2). By (3.10) and $1 / q=1 / r_{i 1}+\cdots+1 / r_{i K}$, we have

$$
\begin{aligned}
\left\|\sup _{t>0}\left|\int \phi_{t,(\cdot)}(x) L_{0}(x) d x\right|\right\|_{L^{q}} & \leq C\left\|\prod_{j=1}^{K}\left[M_{\alpha_{i j} d_{j}}\left(\left|f_{j}\right|^{d_{j}}\right)(\cdot)\right]^{1 / d_{j}}\right\|_{L^{q}} \\
& \leq C \prod_{j=1}^{K}\left\|\left[M_{\alpha_{i j} d_{j}}\left(\left|f_{j}\right|^{d_{j}}\right)(\cdot)\right]^{1 / d_{j}}\right\|_{L^{r_{i j}}} .
\end{aligned}
$$


Since $d_{j}<p_{j}$ and $1 / r_{i j}=1 / p_{j}-\alpha_{i j} / n$, by the above inequality and Lemma 5 we have

$$
\left\|\sup _{t>0}\left|\int \phi_{t,(\cdot)}(x) L_{0}(x) d x\right|\right\|_{L^{q}} \leq C \prod_{j=1}^{K}\left\|f_{j}\right\|_{L^{p_{j}}} .
$$

Finally let us give the weak estimate for $L_{0}$. For any $\lambda>0$, let $\theta_{0}=\lambda$, $\theta_{K}=1$, and $\theta_{1}, \theta_{2}, \ldots, \theta_{K-1}>0$ be arbitrary which be chosen later. Then by (3.11) we get

$$
\begin{aligned}
\left\{x_{0}: \sup _{t>0}\left|\int \phi_{t, x_{0}} L_{0} d x\right|>\lambda\right\} & \\
& \subset \bigcup_{j=1}^{K}\left\{x_{0}:\left[M_{\alpha_{i j} p_{j}}\left(\left|f_{j}\right|^{p_{j}}\right)\left(x_{0}\right)\right]^{1 / p_{j}}>\theta_{j-1} / \theta_{j}\right\} .
\end{aligned}
$$

We now take $\theta_{1}, \theta_{2}, \ldots, \theta_{K-1}>0$ such that

$$
\left(\frac{\theta_{j}}{\theta_{j-1}}\right)^{r_{i j}}=\frac{\prod_{j=1}^{K}\left\|f_{j}\right\|_{p_{j}}^{q}}{\lambda^{q}\left\|f_{j}\right\|_{p_{j}}^{r_{i j}}}, \quad j=1,2, \ldots, K .
$$

By $1 / q=1 / r_{i 1}+1 / r_{i 2}+\cdots+1 / r_{i K}$, we have

$$
\prod_{j=1}^{K}\left(\frac{\theta_{j}}{\theta_{j-1}}\right)=\prod_{j=1}^{K} \frac{\left(\prod_{j=1}^{K}\left\|f_{j}\right\|_{p_{j}}\right)^{q / r_{i j}}}{\lambda^{q / r_{i j}}\left\|f_{j}\right\|_{p_{j}}}=\frac{1}{\lambda} .
$$

Combining (3.15), (3.16) with the weak boundedness of the fractional maximal operator $M_{\alpha}$ (see $\left.[\mathrm{T}]\right)$ and noting that $1 /\left(r_{i j} / p_{j}\right)=1-\left(\alpha_{i j} p_{j}\right) / n$, we have

$$
\begin{aligned}
& \left|\left\{x_{0} \in \mathbb{R}^{n}: \sup _{t>0}\left|\int \phi_{t, x_{0}} L_{0} d x\right|>\lambda\right\}\right| \\
& \leq \sum_{j=1}^{K}\left|\left\{x_{0} \in \mathbb{R}^{n}: M_{\alpha_{i j} p_{j}}\left(\left|f_{j}\right|^{p_{j}}\right)\left(x_{0}\right)>\left(\frac{\theta_{j-1}}{\theta_{j}}\right)^{p_{j}}\right\}\right| \\
& \leq C \sum_{j=1}^{K}\left(\left(\frac{\theta_{j}}{\theta_{j-1}}\right)^{p_{j}} \int_{\mathbb{R}^{n}}\left|f_{j}\left(x_{0}\right)\right|^{p_{j}} d x_{0}\right)^{r_{i j} / p_{j}} \\
& =C \sum_{j=1}^{K}\left(\frac{\theta_{j}}{\theta_{j-1}}\left\|f_{j}\right\|_{p_{j}}\right)^{r_{i j}} \leq \frac{C}{\lambda^{q}} \prod_{j=1}^{K}\left\|f_{j}\right\|_{p_{j}}^{q} .
\end{aligned}
$$


In the last inequality we use (3.16). Thus (3.3) holds for $L_{0}$ and we prove Theorem 1 for the case of all $\alpha_{i j}>0$.

As for the case of $\alpha_{i j}=0$ with some $i, j$, the proof is almost the same as before. In fact, when $\alpha_{i j}=0$, we have $r_{i j}=p_{j}$. By the definition of $T_{\Omega}^{*}$ and Lemmas 1 and 2 we get

$$
\sup _{t>0}\left|T_{\Omega}\left(\eta_{1} f\right)\left(x_{0}\right)\right| \leq T_{\Omega}^{*} f\left(x_{0}\right)+C\left[M\left(|f|^{s^{\prime}}\right)\left(x_{0}\right)\right]^{1 / s^{\prime}} .
$$

Moreover, for any $x$ of satisfying $\left|x-x_{0}\right| \leq t$ and $\Omega \in L^{s^{\prime}}\left(S^{n-1}\right)$

$$
\left|T_{\Omega}\left(\eta_{1} f\right)(x)-T_{\Omega}\left(\eta_{1} f\right)\left(x_{0}\right)\right| \leq C\left[M\left(|f|^{s^{\prime}}\right)\left(x_{0}\right)\right]^{1 / s^{\prime}}
$$

By (3.18), (3.19) and Theorem B and using the same method above, we may obtain the conclusion of Theorem1 for this case. Here we omit the details.

\section{§4. Proof for Theorem 2}

Now we apply the conclusion of Theorem 1 (taking $K=N=2$ ) to give the proof of Theorem 2 . In fact, it is easy to verify that

$$
\begin{aligned}
\left\|T_{\Omega, \alpha, b}(f)\right\|_{q} & =\sup _{g}\left|\int_{\mathbb{R}^{n}} g(x)\left[b(x) T_{\Omega, \alpha} f(x)-T_{\Omega, \alpha}(b f)(x)\right] d x\right| \\
& =\sup _{g}\left|\int_{\mathbb{R}^{n}} b(x)\left[g(x) T_{\Omega, \alpha} f(x)-f(x) T_{\Omega, \alpha}^{\prime} g(x)\right] d x\right|,
\end{aligned}
$$

where the supremun is taken over all functions $g(x) \in L^{q^{\prime}}$ with $\|g\|_{q^{\prime}} \leq 1$. Moreover, $T_{\Omega, \alpha}^{\prime}$ denotes the adjoint operator of $T_{\Omega, \alpha}$.

On the other hand, if replacing the singular integral operator by the identical operator in the $K$-linear operator $L_{\Omega, \alpha}(\vec{f})$, then the conclusion of Theorem 2 still holds. Note that

$$
\int_{\mathbb{R}^{n}}\left(g(x) T_{\Omega, \alpha} f(x)-f(x) T_{\Omega, \alpha}^{\prime} g(x)\right) d x=0 .
$$

Hence, by the conditions of Theorem 2 and (4.2), and using the result of Theorem 1, we have

$$
\left\|g T_{\Omega, \alpha} f-f T_{\Omega, \alpha}^{\prime} g\right\|_{H^{1}} \leq C\|f\|_{p}\|g\|_{q^{\prime}}
$$


Since $b(x) \in B M O$ and $\left(H^{1}\right)^{*}=B M O$, by (4.1), (4.3) and the choice of $g$, we get

$$
\left\|T_{\Omega, \alpha, b}(f)\right\|_{q} \leq \sup _{g}\|b\|_{B M O}\left\|g T_{\Omega, \alpha} f-f T_{\Omega, \alpha}^{\prime} g\right\|_{H^{1}} \leq C\|b\|_{B M O}\|f\|_{p} .
$$

Thus, we obtain the conclusion of Theorem 2 .

Acknowledgement. The authors would like to express their deep gratitude to the referee for his very valuable comments and suggestions. Moreover, Yong Ding would like to thank Professor Akihiko Miyachi for giving him the preprint of the paper [Mi].

\section{REFERENCES}

[CZ] A. Calderón and A. Zygmund, A note on the interpolation of Sublinear operators, Amer. J. Math., 78 (1956), 282-288.

[Cha] S. Chanillo, A note on commutators, Indiana. Univ. Math. J., 31 (1982), 7-16.

[CWW] S. Chanillo, D. Watson and R. L. Wheeden, Some integral and maximal operators related to star-like, Studia Math., 107 (1993), 223-255.

[Che] L. K. Chen, On a singular integral, Studia Math., 85 (1987), 61-72.

[CG] R. Coifman and L. Grafakos, Hardy spaces estimates for multilinear operators I, Rev. Math. Iber., 8 (1992), 45-68.

[D] Y. Ding, Weak type bounds for a class of rough operators with power weights, Proc. Amer. Math. Soc., 125 (1997), 2939-2942.

[DL1] Y. Ding and S. Z. Lu, The $L^{p_{1}} \times L^{p_{2}} \times \cdots \times L^{p_{k}}$ boundedness for some rough operators, Jour. Math. Anal. Appl., 203 (1996), 166-186.

[DL2] Y. Ding and S. Z. Lu, Weighted norm inequalities for fractional integral operators with rough kernel, Canad. J. Math., 50 (1998), 29-39.

[DL3] Y. Ding and S. Z. Lu, Homogeneous fractional integrals on Hardy spaces, Tôhôku Math. J., 52 (2000), 153-162.

[DL4] Y. Ding and S. Z. Lu, Hardy spaces estimates for a class of multilinear homogeneous operators, Science in China (A), 42 (1999), 1270-1278.

[FS] R. Fefferman and F. Soria, The Weak space $H^{1}$, Studia Math., 85 (1987), 1-16.

[G] L. Grafakos, Hardy spaces estimates for multilinear operators II, Rev. Math. Iber., 8 (1992), 69-92.

[KW] D. Kurtz and R. L. Wheeden, Results on weighted norm inequalities for multipliers, Trans. Amer. Math. Soc., 255 (1979), 343-362.

[Lu] S. Z. Lu, Four lectures on real $H^{p}$ spaces, World Scientific Publishing Co. Pte. Ltd., 1995.

[Mi] A. Miyachi, Hardy spaces estimates for the product of singular integrals, Canad. J. Math., 52 (2000), 281-311.

[MW] B. Muckenhoupt and R. L. Wheeden, Weighted norm inequalities for singular and fractional integrals, Trans. Amer. Math. Soc., 161 (1971), 249-258. 
[S] E. M. Stein, Harmonic Analysis: Real-Variable Methods, Orthogonality, and Ocillatory Integrals, Princeton Univ. Press, Princeton, N.J., 1993.

[T] A. Torchinsky, Real-Variable Methods in Harmonic Analysis, Academic Press, 1986.

Yong Ding

Department of Mathematics

Beijing Normal University

Beijing 100875

China

dingy@bnu.edu.cn

Shanzhen Lu

Department of Mathematics

Beijing Normal University

Beijing 100875

China

lusz@bnu .edu.cn 